\title{
Analysis of the Influence of Mill Helix Angle on Chatter Stability
}

\author{
M. Zatarain ${ }^{1}(2)$, J. Muñoa ${ }^{2}$, G. Peigné ${ }^{3}$, T. Insperger ${ }^{4}$ \\ ${ }^{1}$ Tekniker Fundazioa, Dept. of Mechatronics and Precision Engineering, Eibar, Basque Country, Spain \\ ${ }^{2}$ Ideko Technological Centre, Elgoibar, Basque Country, Spain \\ ${ }^{3}$ Institut de Recherche en Communication et Cybernétique de Nantes, Ecole Centrale de Nantes, France \\ ${ }^{4}$ Budapest University of Technology and Economics, Dept. of Applied Mechanics, Hungary
}

\begin{abstract}
Current methods for estimating chatter stability limits for milling do not consider the influence of the helix angle and the consequent phase lag between the forces appearing at different sections of the mill. Budak and Altintas' multifrequency solution is extended to include the helix effect, and results are compared with results of semi-discretization and experiments.

As a conclusion, the helix has an important influence on the areas of added lobes (flip lobes), while the influence on the traditional lobes is negligible. Flip lobes become closed curves separated by horizontal lines where the depth of cut equals a multiple of the helix pitch.
\end{abstract}

Keywords: End milling, Stability, Dynamics

\section{INTRODUCTION}

Chip removing machine tools find a productivity limitation due to the appearance of self excited vibration. The basics of that problem is well known since a long time ago.

Mathematical models able to represent the behaviour of milling process are much more complex than those representing continuous processes. Nevertheless, during the last years important advance was done in obtaining good theoretical approaches for milling stability.

These new mathematical models permitted the identification of a kind of instability not described previously, which was called as 'period doubling' or 'flip'. Mathematically this phenomenon was shown to exist, and it was also found in experiments, but no engineering explanation of it was given. This kind of instability is associated with repeated impacts at fixed frequencies, typically twice the natural frequency of the system.

The use of helicoidal mills in end milling processes gives rise to a less discontinuous milling process. Because of that, it could be possible that the helix angle has a positive effect on the repetitive impact driven instability (flip). This work aims to analyse this effect from a multifrequency analysis approach, and validate the analysis by semidiscretization and experimentation.

\section{STATE OF THE ART}

Tobias [1] and Tlusty [2], almost simultaneously, published papers explaining chatter as a regenerative phenomenon. Later, Merrit [3] presented the problem as a feed back loop, which clarified a lot the formulation.

For many years these basic approaches were used, by reducing the dynamics of the machine to an equivalent single degree of freedom system.

In 1995 Altintas and Budak [4] presented the method which enables working with several degree of freedom models. At the same time, they analysed the geometrical nonlinearities of the milling process, obtained an approach to the solution by using a Fourier series development of the directional factors and solved the system by considering the zero order term only.

Later, Budak and Altintas [5] worked out the system by considering several terms of the Fourier development, which gives rise to solutions very close to those obtained by using the fundamental term only.
The single frequency approach has been shown to be very precise, but when radial immersion of the mill is small, the existence of additional stability lobes was found. Davies et. al. [6] used a discrete map model for highly interrupted milling processes, where the time in the cut is infinitesimal and the cutting process is modelled as an impact. Insperger and Stépán [7] used an analytical approximation method called Fargue-method. Later, they developed the technique of semi-discretization [8]. Bayly et. al. [9] obtained similar results using temporal finite elements.

Merdol and Altintas [10] showed that the multifrequency resolution is also able to represent accurately the flip instability phenomenon.

Just a few papers analysed the chatter in milling with inclusion of the effect of the helix angle. Balachandran et al. [11] performed time domain calculations, while Gradišek et. al. [12] worked by the semi-discretization method. Nevertheless, none of them analysed the effect of different helix angles. Some papers [12][13] presented some instability regions with 'lenticular' shape, but they were not associated with the helix angle of the mill.

\section{REPETITIVE IMPACT DRIVEN CHATTER}

The most usually found chatter mechanism is that of feed back of vibration from the marks left by the tool on the part in the previous pass. This kind of vibration is called 'regenerative chatter'. When the marks have a phase lag with the relative vibration between tool and part there is the possibility that the additional force produced by the marks increments the energy of the vibratory system (force and speed in phase) or decrements it (force and speed in counter phase).

Instability due to flip is characterized by a cutting frequency exactly equal to twice the chatter vibration frequency. A harmonic force of frequency double the vibration frequency will not produce any change in the vibratory energy, and, therefore, a self excited vibration could not be explained like that. However, short, repetitive impacts with a frequency twice the vibration frequency, and amplitude dependent on the amplitude of the vibration could increase the vibratory energy.

Figure 1 shows the effect of increasing the vibratory energy by repetitive impacts: The red line represents system vibration, while the blue bars represent the impulse produced by the impact once every revolution. That 
impulse is proportional to the displacement, and produces a momentum increment. The result is that the amplitude of vibration increases continuously.

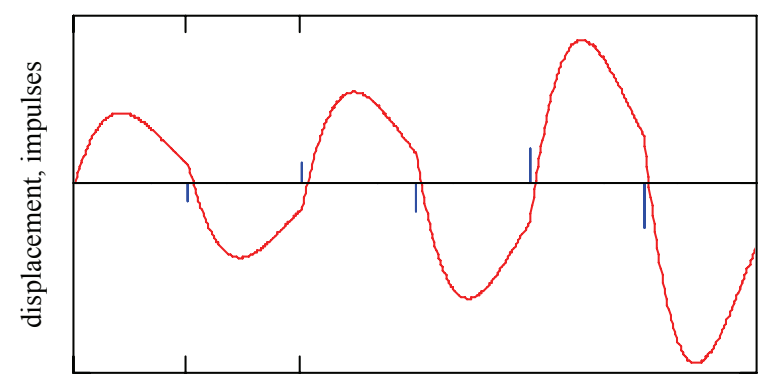

time

Figure 1: Repetitive impact driven vibration.

Repetitive impact driven instability phenomenon requires impulsive type of forces. If harshness could be reduced, a lower tendency to instability could be expected.

\section{MULTIFREQUENCY THEORY INCLUDING THE EFFECT OF HELIX ANGLE}

Milling process stability analysis in the frequency domain for straight fluted mills was presented in detail in several papers [4] [5] [10]. To reduce the mathematics involved to the minimum, here only a draft of the modifications to be done on those formulations in order to include the helix angle effect will be shown.

Again, with the goal of simplifying explanation, an $X$ direction single degree of freedom system will be used. The development could be easily extended to other degrees of freedom by repeating the same procedure explained in the aforementioned papers.

Instantaneous chip thickness $h$ for a particular flute of the mill at angular position $v$ with respect to the $Y$ axis is:

$h=\sin (v) \cdot\left(a_{p}+\delta(t)-\delta(t-\tau)\right)$

where $\delta=\delta_{x}$ is the relative instantaneous displacement between tool and part due to system deformation, $a_{p}$ is the feed per tooth, $t$ is time and $\tau$ tooth pass period.
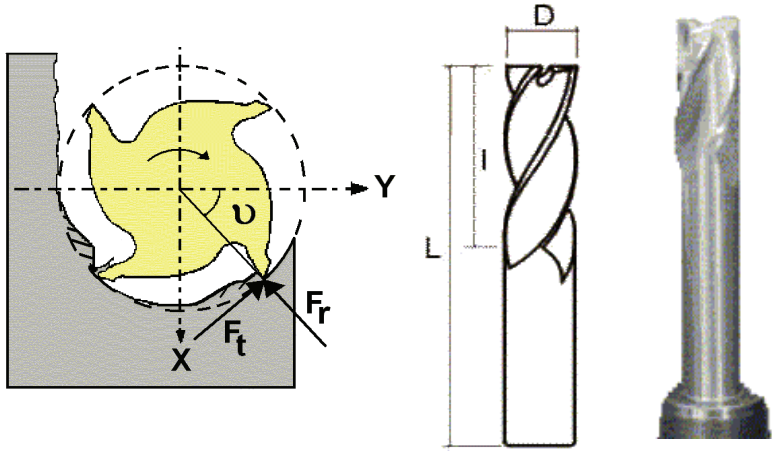

Figure 2: Milling forces and pictures of helical mill.

Assuming a linear cutting force model, the force can be calculated as

$\left\{\begin{array}{l}f_{x}(t) \\ f_{y}(t) \\ f_{z}(t)\end{array}\right\}=\left[\begin{array}{ccc}-c & -s & 0 \\ s & -c & 0 \\ 0 & 0 & -1\end{array}\right]\left\{\begin{array}{c}1 \\ \rho_{r} \\ \rho_{a}\end{array}\right\} \cdot b \cdot k_{t} \cdot s \cdot\left(a_{p}+\delta(t)-\delta(t-\tau)\right)(2)$

where $k_{t}$ is the specific tangential force, $\rho$ means the ratio of passive force to tangential force, subindices $t, r$, a refer to the tangential, radial and axial directions, and $c, s$ are respectively the cosines and sines of the angular position $v$ of the flute.

From the three force components, only that in the $X$ direction is of interest, as a single degree of freedom system was assumed in that direction. So:

$$
\begin{aligned}
f(t) & =-\left(c \cdot s+\rho_{r} \cdot s^{2}\right) \cdot b \cdot k_{t} \cdot\left(a_{p}+\delta(t)-\delta(t-\tau)\right) \\
& \equiv-\psi(v) \cdot b \cdot k_{t} \cdot\left(a_{p}+\delta(t)-\delta(t-\tau)\right)
\end{aligned}
$$

where $\psi(v)$ is the instantaneous directional factor.

In this equation, the term $a_{p}$ is only responsible for the forced vibration and has no responsibility on the system stability. So, it is possible to delete it from the equation.

If the directional factor is developed in a Fourier series we obtain

$\psi(v(t))=\psi_{0}+\psi_{-1} \cdot e^{-j . \Omega . t}+\psi_{1} \cdot e^{j . \Omega \cdot t}+\psi_{-2} \cdot e^{-j 2 . \Omega . t}+\ldots$

where $\Omega$ is the tooth pass frequency.

If the approach by only the first term is used, the single frequency solution is obtained. That solution produces correct results for large radial immersions of the mill, but for small radial immersions more terms of the Fourier series have to be used. Those terms allow the flip lobes, or repetitive impact driven lobes, to appear.

If the system has a constant amplitude vibration with frequency $\omega$ at the stability limit, then substitution of the instantaneous directional factor by its Fourier development gives:

$f=-\left(\sum_{k=-\infty}^{\infty} \psi_{1} \cdot e^{j \cdot \Omega \cdot t}\right) \cdot b \cdot k_{t} \cdot\left(1-e^{-j \cdot \omega \cdot \tau}\right) \cdot \Delta \cdot e^{j \cdot \omega \cdot t}$

where $\Delta$ is the amplitude of the harmonic displacement.

Equation (5) shows that a vibration of frequency $\omega$ produces forces with frequencies $\omega, \omega+\Omega, \omega-\Omega, \omega+2 \Omega$, $\omega-2 \Omega$, etc. These forces will give rise to displacements at the same frequencies. Therefore, it is necessary to work out a system of simultaneous vibrations at all those frequencies. If only the terms corresponding to frequencies $\omega, \omega-\Omega, \omega+\Omega$ are included, then:

$$
\begin{aligned}
& \left\{\begin{array}{c}
F_{\omega} \\
F_{\omega-\Omega} \\
F_{\omega+\Omega}
\end{array}\right\}=-b \cdot k_{s} \cdot\left(1-e^{-j \cdot \omega \cdot \tau}\right)\left[\begin{array}{ccc}
\psi_{0} & \psi_{1} & \psi_{-1} \\
\psi_{-1} & \psi_{0} & \psi_{-2} \\
\psi_{1} & \psi_{2} & \psi_{0}
\end{array}\right]\left\{\begin{array}{c}
\Delta_{\omega} \\
\Delta_{\omega-\Omega} \\
\Delta_{\omega+\Omega}
\end{array}\right\} \\
& \delta(t)=\Delta_{\omega} \cdot e^{j \cdot \omega \cdot t}+\Delta_{\omega+\Omega} \cdot e^{j \cdot(\omega+\Omega) \cdot t}+\Delta_{\omega-\Omega} \cdot e^{j \cdot(\omega-\Omega) \cdot t} \\
& f(t)=F_{\omega} \cdot e^{j \cdot \omega \cdot t}+F_{\omega+\Omega} \cdot e^{j \cdot(\omega+\Omega) \cdot t}+F_{\omega-\Omega} \cdot e^{j \cdot(\omega-\Omega) \cdot t}
\end{aligned}
$$

Dynamic behaviour of the system is represented by

$$
\begin{aligned}
& \left\{\begin{array}{c}
\Delta_{\omega} \\
\Delta_{\omega-\Omega} \\
\Delta_{\omega+\Omega}
\end{array}\right\}=[\mathrm{H}]\left\{\begin{array}{c}
F_{\omega} \\
F_{\omega-\Omega} \\
F_{\omega+\Omega}
\end{array}\right\} \\
& \text { with }[\mathrm{H}]=\left[\begin{array}{lll}
H(\omega) & & \\
& H(\omega-\Omega) & \\
& & H(\omega+\Omega)
\end{array}\right]
\end{aligned}
$$

where $H(\omega)$ is the transfer function between force and displacement at frequency $\omega$. Substituting in (6): 


$$
\begin{aligned}
\left\{\begin{array}{c}
F_{\omega} \\
F_{\omega-\Omega} \\
F_{\omega+\Omega}
\end{array}\right\} & \left.=-b \cdot k_{s} \cdot\left(1-e^{-j \cdot \omega \cdot \tau}\right)\left[\begin{array}{ccc}
\psi_{0} & \psi_{1} & \psi_{-1} \\
\psi_{-1} & \psi_{0} & \psi_{-2} \\
\psi_{1} & \psi_{2} & \psi_{0}
\end{array}\right][\mathrm{H}]\right\}\left\{\begin{array}{c}
F_{\omega} \\
F_{\omega-\Omega} \\
F_{\omega+\Omega}
\end{array}\right\} \equiv \\
& \equiv-b \cdot k_{s} .\left(1-e^{-j \cdot \omega \cdot \tau}\right) \cdot[\mathrm{M}]\left\{\begin{array}{c}
F_{\omega} \\
F_{\omega-\Omega} \\
F_{\omega+\Omega}
\end{array}\right\}
\end{aligned}
$$

Stability is analysed by calculating the eigenvalues of this system [4] [5]. The eigenvalues of matrix $M$, multiplied by $b . k s$ must equal -1 . In the practice, it is necessary to vary the frequency $\omega$ until the imaginary part of the eigenvalue is zero, and $b$ is calculated so that $b . k s . \lambda=-1$ is fulfilled.

The development up to now did not consider the angular shift between the planes of the tool at different heights, that is, the effect of the helix. That shift angle causes the highest planes get into contact later than the lowest ones (right handed helix mills). Because of that, to include the axial position of each plane, equation (1) becomes:

$$
\begin{aligned}
h(z) & =\sin (v(z)) \cdot\left(h_{t}+\delta(t)-\delta(t-\tau)\right)= \\
& =\sin \left(v_{0}-2 \cdot \pi \frac{z}{N \cdot p} \cdot\right) \cdot\left(h_{t}+\delta(t)-\delta(t-\tau)\right)
\end{aligned}
$$

where $p=D \pi /(N \tan (\eta))$ is the helix pitch of the mill with $N$ the number of flutes, $D$ diameter of the tool and $\eta$ helix angle, and $v_{0}(t)$ is the angle determined by the flute with the $Y$ axis at the lowest plane (at $z=0$ ).

Total force should now be calculated by an integration of the force at each plane along $Z$ direction. In that way:

$$
\begin{aligned}
& f(t)=-\int_{0}^{b}\left(c . s+k_{r} \cdot s^{2}\right) \cdot k_{s} \cdot\left(h_{t}+\delta(t)-\delta(t-\tau)\right) \cdot d z \\
& c=\cos \left(v_{0}(t)-2 \cdot \pi \cdot \frac{z}{N \cdot p}\right) \quad s=\sin \left(v_{0}(t)-2 \cdot \pi \cdot \frac{z}{N \cdot p}\right)
\end{aligned}
$$

Thus, Fourier development of directional factor should be performed by an integration in the $Z$ direction. Then:

$$
\left\{\begin{array}{c}
F_{\omega} \\
F_{\omega-\Omega} \\
F_{\omega+\Omega}
\end{array}\right\}=-b . k_{s} .\left(1-e^{-j . \omega \cdot \tau}\left[\begin{array}{ccc}
\psi_{0}^{*} & \psi_{1}^{*} & \psi_{-1}^{*} \\
\psi_{-1}^{*} & \psi_{0}^{*} & \psi_{-2}^{*} \\
\psi_{1}^{*} & \psi_{2}^{*} & \psi_{0}^{*}
\end{array}\right][H]\left\{\begin{array}{c}
F_{\omega} \\
F_{\omega-\Omega} \\
F_{\omega+\Omega}
\end{array}\right\}\right.
$$

with $\psi_{k}^{*}=\psi_{k} \cdot \frac{1-e^{-j \cdot k \cdot \frac{2 \cdot \pi \cdot b}{p}}}{j \cdot k \cdot \frac{2 \cdot \pi \cdot b}{p}} \equiv \psi_{k} \cdot g_{k}$

where $\psi_{k}$ is the $k^{\text {th }}$ Fourier term of directional factor when helix angle is not considered, and $\psi_{k}^{*}$ is the same Fourier term when the effect of the helix angle is included.

Equation (11) could be worked out in the same way as the traditional system, but the problem appears that the directional factors vary with axial immersion $b$. An iterative solution, consisting of assuming $b$, calculating directional factors, solving the system to get $a$ new $b$ value, and starting again, produces good results most of the times.

Zero order directional factor agrees with the usual one, as function $g_{0}$ (defined at equation (12)) equals 1 . For other orders, if the evolution of $g_{k}$ is represented against the axial immersion of the mill, Figure 3 is obtained.

Figure 3 shows that when immersion approaches mill helix pitch, directional factors of orders 1 and larger will cancel, what means that the system is reduced to the fundamental solution presented in [4]. For other immersions, the magnitude of directional factors is reduced, what gives rise to a reduced tendency to repetitive impact driven chatter (flip).

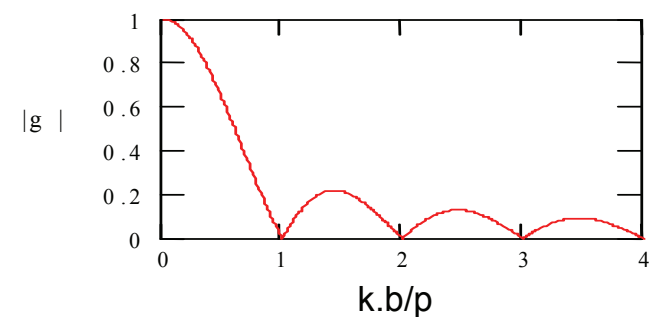

Figure 3: Evolution of $\left|g_{k}\right|$.

\subsection{Theoretical results based on multifrequency}

The theory developed was applied to a simple case of a single degree of freedom system described in Figure 6. The theoretical results are shown in Figure 4. The blue line indicates the result for a straight flute mill, while red lines and isles indicate the limit depths for the helical mill.

Immediatly noticeable is that for helical mills there are some instability isles in the flip region, and the next limit (red continuous line) is practically coincident with the zero order solution. There is no instability due to flip when the depth of cut is a multiple of the mill helix pitch.

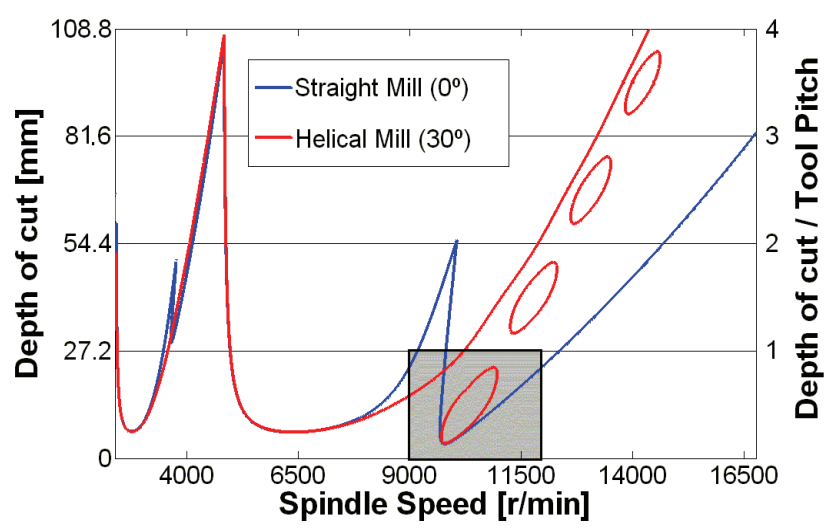

Figure 4: Instability areas for helical mill.

Straight and helical mill limits are practically coincident except for the flip region. There, for the lowest speed values, the blue line and red first isle coincide, but for higher speeds the limit depth for helical mill is larger.

The results for multifrequency model were checked by a previously mentioned model, developed by using the method of semi-discretization [8]. Stability limits obtained by the two methods are exactly the same, which guarantees that the theoretical results are correct.

\subsection{Experimental validation}

A set of tests was performed on an aluminium 7075 T6 part on a fixture with high flexibility in $Y$ direction. The feed direction coincides with $X$ axis. Figure 5 details the main characteristics of the test.

The tests were oriented to verify the presence of the first unstable island. Therefore the cutting tests were carried out in the range $9000-11750 \mathrm{r} / \mathrm{min}$ (shadowed box in Figure 4). Specific force parameters were identified in a mechanistic way to be $k_{t}=804.3 \mathrm{~N} / \mathrm{mm}^{2}, k_{r}=331 \mathrm{~N} / \mathrm{mm}^{2}$.

Low diameter end mills can have an important influence on chatter stability. For that reason, the mill selected was mounted on the tool holder with a free length as short as possible. Limit depth of cut due to the mill flexibility was estimated to be sufficiently larger than that due to the flexible fixture. 


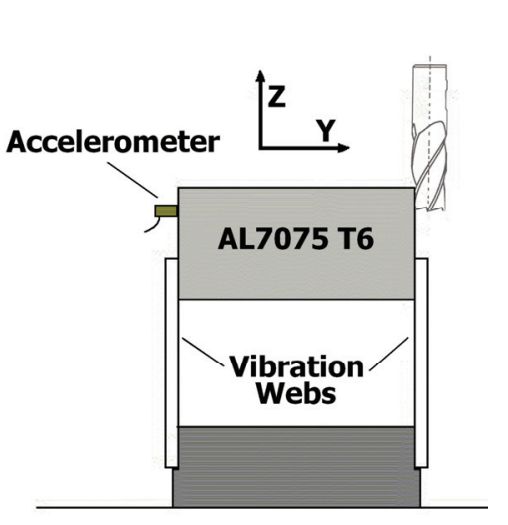

Dynamic parameters: $\omega=319.375 \mathrm{~Hz}$. $\mathrm{K}=21.6 \mathrm{~N} / \mu \mathrm{m}$. $\xi=1.96 \%$

Y direction

Tool characteristics: $\mathrm{N}=4$

Diameter $=20 \mathrm{~mm}$ Helix $=30^{\circ}$

Pitch $=27.2 \mathrm{~mm}$

Cutting Conditions:

Feed $=0.1 \mathrm{~mm} /$ flute

Feed Direction $=\mathrm{X}+$

Radial immer. $=1 \mathrm{~mm}$ (Down milling)

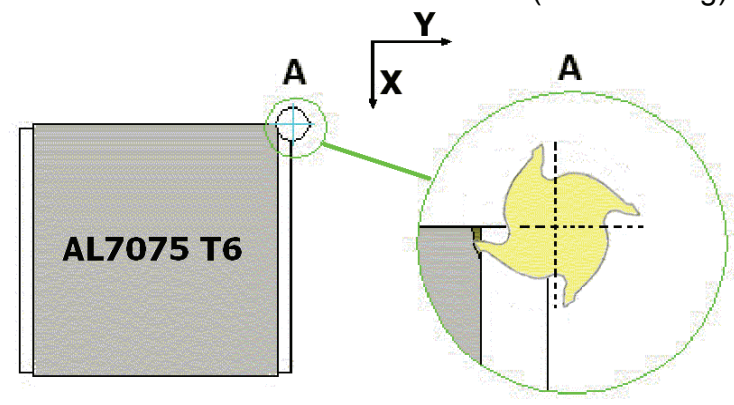

Figure 5: Aluminium part and flexible fixture.

As the tool had 4 flutes, frequency of chatter due to period doubling (flip) was two times rotating speed, where there is strong forced vibration. So, it is not easy to objectively assess the presence of chatter from the vibration measured. For that reason, Figure 6 describes the vibration level measured during the tests by means of isolines. The theoretical stability limits (red lines), and the stability limits for an equivalent mill with straight flutes (blue lines) are also included in the figure. The vibration level isolines have the same island shape found in the theoretical approach. We can conclude that the experiments fully validate the theory developed. The presence of an instability island was correlated with unstable cuts with a high vibration level.

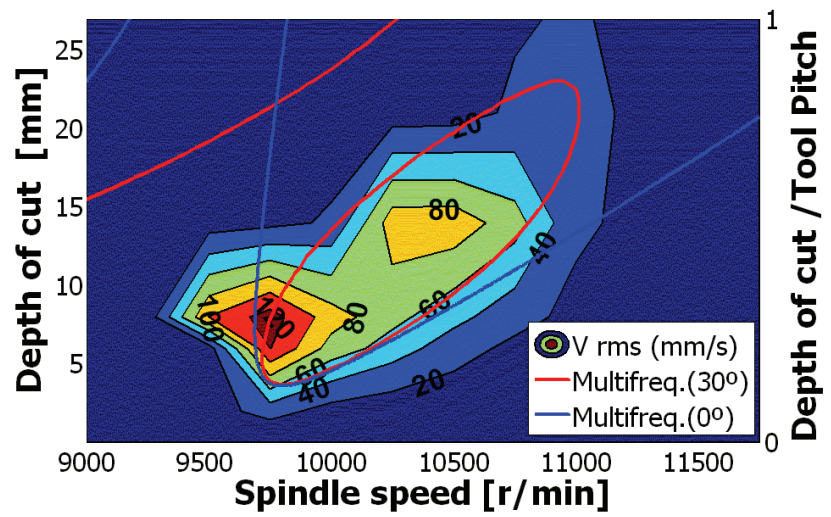

Figure 6: Experimental measurements versus stability lobes.

\section{CONCLUSIONS}

The theory for inclusion of helix angle effect on Budak and Altintas' multifrequency chatter stability models [5] was developed. The helix angle was shown to reduce the importance of higher order harmonics. Therefore, zero order solution can be valid for almost all circumstances.

The analysis showed that helix angle of the mill can have an important role on instability due to repetitive impact driven chatter (flip). If the axial depth of cut is equal to a multiple of the helix pitch, only zero order directional term remains, since the helix averages the variation of the directional factors. Cancelling of harmonics of the directional factor prevent the appraising of added lobes

As a consequence, period doubling instability areas are closed islands, separated by horizontal lines where the depth of cut equals a multiple of the mill helix pitch length.

\section{ACKNOWLEDGMENTS}

The authors acknowledge the collaboration of Patxi Aristimuño, from Mondragon University, in the tests.

\section{REFERENCES}

[1] Tobias, S.A., Fiswick, W., 1958, Theory of Regenerative Machine Tool Chatter, Engineering, London, 258.

[2] Tlusty, J., Polacek, M., 1957, Beispiele der behandlung der selbsterregten Schwingung der Werkzeugmaschinen FoKoMa, Hanser Verlag, Munchen.

[3] Merritt, H., 1965, Theory of Self-Excited Machine Tool Chatter, Journal of Engineering for Industry, 87:447-454.

[4] Altintas, Y., Budak, E., 1995, Analytical Prediction of Stability Lobes in Milling, Annals of the CIRP, 44/1:357-362.

[5] Budak, E., Altintas, Y., 1998, Analytical Prediction of Chatter Stability Conditions for Multidegree of Freedom Systems in Milling. Part I: General Formulation, Part II: Application of the General formulation to Common Milling Systems", Transactions of the ASME, 120:22-36.

[6] Davies, M., Pratt, J.R., Dutterer, B.S., Burns, T.J., 2000 , The stability of low radial immersion milling, Annals of the CIRP, 49/1:37-40.

[7] Insperger, T., Stépán, G., 2000, Stability of High Speed Milling, in Proceedings of the 2000 ASME International Engineering Congress and Exposition, Orlando, Florida, AMD - 241:119-123.

[8] Insperger, T., Stépán, G., 2004, Updated SemiDiscretization Method for Periodic Delay-Differential Equations with Discrete Delay, International Journal of Numerical Methods in Engineering, 61/1:117-141.

[9] Bayly, P.V., Halley, J.E., Mann, B.P., Davies, M.A., 2003, Stability of Interrupted Cutting by Temporal Finite Element Analysis, Journal of Manufacturing Science and Engineering, 125:220-225.

[10] Merdol, S.D., Altintas, Y., 2004, Multi Frequency Solution of Chatter Stability for Low Immersion Milling, Journal of Manufacturing Science and Engineering, 126:459-466.

[11] Balachandran, B., Zhao, M. X., 2000, A Mechanics Based Model for Study of Dynamics of Milling Operations, Meccanica, 35/2:89-109.

[12] Szalai, R., Stépán, G., 2003, Stability boundaries of high-speed milling corresponding to period doubling are essentially closed curves, Proceedings of IMECE2003 ASME International Mechanical Engineering Congress and R\&D Expo, Washington D.C., IMECE2003-42122.

[13] Govekar,E., Gradisek, J., Kalveram, M., Insperger, T., Weinert, K., Stepan, G., Grabec, I., 2005, On Stability and Dynamics of Milling at Small Radial Immersion, Annals of the CIRP, 54/1:357-362. 